\title{
DRENAGEM ABERTA VERSUS DRENAGEM PERCUTÂNEA NO TRATAMENTO DA PIOMIOSITE TROPICAL. ESTUDO PROSPECTIVO E RANDOMIZADO
}

\author{
OPEN DRAINAGE VERSUS PERCUTANEOUS DRAINAGE IN THE TREATMENT \\ OF TROPICAL PYOMYOSITIS. PROSPECTIVE AND RANDOMIZED STUDY
}

Evandro Pereira Palacio', Nívea Gitahy Rizzi², Gustavo Serra Reinas², Melvis Michiuti Júnior², Alcides Durigan Júnior ${ }^{3}$, Roberto Ryuiti Mizobuchi', Ricardo Hideki Yanasse', Marcos Vinícius Muriano da Silva', Rodrigo Borsatto Branco ${ }^{5}$, José Antônio Galbiatti ${ }^{6}$

\section{RESUMO}

Objetivo: Comparar, através de estudo prospectivo e randomizado, os resultados do tratamento da piomiosite tropical (PT) através da drenagem percutânea dos abscessos aos da drenagem cirúrgica aberta dos mesmos. Métodos: Foram incluídos neste estudo 25 pacientes com PT, grau II de Chiedozi. Os pacientes foram randomizados em dois grupos: grupo $A(n=13)$, tratados com antibioticoterapia e drenagem aberta dos abscessos e grupo B ( $n=12)$, tratados com antibioticoterapia e drenagem percutânea dos mesmos. Resultados: A idade média no grupo A foi de 35,3 $\pm 19,2$ anos e, no grupo $B$, de $30,1 \pm 9$ anos $(p=0,41)$. No grupo A, oito pacientes (61,5\%) eram do sexo feminino e cinco do masculino (38,5\%); no grupo B, três eram do sexo feminino (25\%) e nove do masculino $(75 \%)(p=0,11)$. O Staphylococcus aureus foi o microorganismo mais frequentemente encontrado (72\%). O tempo médio de internação no grupo A foi de $12,7 \pm 2,1$ dias e, no grupo $\mathrm{B}$, de $10,6 \pm 1,6$ dias $(\mathrm{p}=0,01)$. O tempo médio de antibiótico no grupo A foi de 12,2 $\pm 2,3$ dias e, no grupo B, de 10,1 $\pm 1,5$ dias ( $p=0,02)$. Conclusão: A drenagem percutânea dos abscessos associada à antibioticoterapia constituiu-se em método eficaz para o tratamento da piomiosite tropical grau II, diminuindo o tempo de antibioticoterapia e de internação hospitalar dos pacientes.

Descritores - Piomiosite; Miosite; Staphylococcus aureus; Abscesso

\section{ABSTRACT}

Objective: To compare, by means of a prospective and randomized study, the treatment outcomes of Tropical Pyomyositis (TP) through open drainage of abscesses versus percutaneous surgical drainage. methods: 25 patients with TP (Chiedozi stage II) were randomized into two groups: Group A $(n=13)$, treated with antibiotics and open drainage of the abscesses, and Group $B(n=12)$, treated with antibiotics and percutaneous drainage. Results: The mean age was 35.3 years $( \pm 19.2)$ in Group A and 30.1 years in Group $B( \pm 9)(p=0.41)$. There were eight female (61.5\%) and five male (38.5\%) patients in Group A; in Group B three were female (25\%) and nine (75\%) male ( $p=0.11)$. Staphylococcus aureus was the most frequent agent found (72\%). The mean hospitalization period in Group A was 12.7 days ( \pm 2.3$)$, and in Group B, 10.6 days $( \pm 1.6)(p=0.01)$. The mean antibiotics treatment period in Group A was 12.2 days ( \pm 2.3$)$, and 10.1 days $( \pm 1.5)$ in Group B $(p=0.02)$. Conclusion: The percutaneous drainage of the abscesses is an efficient treatment method for the Tropical Pyomyositis, with a shorter period of hospitalization and antibiotics usage.

Keywords - Pyomyositis; Myositis; Staphylococcus aureus; Abscess

\footnotetext{
1 - Professor Assistente do Departamento de Ortopedia e Traumatologia da Faculdade de Medicina de Marília - Marília/SP.

2 - Médico Residente do Departamento de Ortopedia e Traumatologia da Faculdade de Medicina de Marília - Marília/SP.

3 - Chefe do Grupo de Ombro e Cotovelo do Departamento de Ortopedia e Traumatologia da Faculdade de Medicina de Marília - Marília/SP.

4 - Chefe do Serviço. Chefe do Grupo de Joelho do Departamento de Ortopedia e Traumatologia da Faculdade de Medicina de Marília - Marília/SP.

5 - Acadêmico do $5^{\circ}$ ano do Curso de Medicina da Faculdade de Medicina de Marília - Marília/SP.

6 - Chefe do Grupo de Cirurgia da Mão do Departamento de Ortopedia e Traumatologia da Faculdade de Medicina de Marília - Marília/SP.
}

Trabalho realizado no Departamento de Ortopedia e Traumatologia da Faculdade de Medicina de Marília/Famema - Marília/SP.

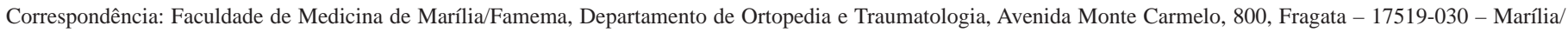
SP, Brasil. E-mail: palacio@famema.br 


\section{INTRODUÇÃO}

A piomiosite tropical (PT), também conhecida como piomiosite primária, miosite infecciosa, miosite piogênica, miosite supurativa ou miosite bacteriana ${ }^{(1)}$, é uma enfermidade com alta incidência em países de clima tropical; contudo, cada vez mais está sendo relatada em países de climas temperados ${ }^{(2-13)}$. Causada em mais de $90 \%$ dos casos pelo Staphylococus aureus ${ }^{(2-20)}$, esta enfermidade tem recebido pouca atenção da literatura especializada, principalmente ortopédica, mesmo face às graves sequelas descritas na insuficiente literatura disponível e bancos de dados existentes. Tais sequelas, quando não tratadas de forma imediata e conveniente, frequentemente culminam em graves complicações para os pacientes como necrose muscular, síndrome compartimental, abscessos cerebrais, insuficiência renal, septicemia e óbito ${ }^{(6,8-10,12,13,15,16,21)}$.

A PT pode acometer indivíduos de qualquer faixa etária, com um ligeiro predomínio na segunda e terceira décadas de $\operatorname{vida}^{(2-23)}$. A inespecificidade inicial dos sintomas, como: febre, dor e ligeira enduração do músculo afetado, como descrito por Chiedozi ${ }^{(5)}$, além das poucas evidências de um processo infeccioso em instalação, levam, não raro, a atraso ou erro diagnóstico.

O diagnóstico da PT é essencialmente clínico. Exames como ultrassonografia, tomografia computadorizada e ressonância magnética têm seu espaço, sobretudo quando a musculatura afetada encontra-se em planos profundos ou, ainda, com a finalidade de se definir possíveis diagnósticos diferenciais; contudo, a falta de qualquer um destes exames não pode impedir ou retardar o diagnóstico e o início do tratamento, ainda que de forma empírica ${ }^{(1-24)}$.

$\mathrm{O}$ tratamento proposto para a PT diz respeito à efetividade do tratamento isolado com antibióticos, quando em seu estágio inicial e, quando diagnosticada tardiamente, da drenagem aberta dos abscessos associada à ampla cobertura antibiótica.

O objetivo deste trabalho é comparar, através de estudo prospectivo e randomizado, os resultados do tratamento da PT através de antibioticoterapia e drenagem percutânea dos abscessos aos da terapia convencional, com antibióticos associados à drenagem cirúrgica aberta dos mesmos.

\section{MÉTODOS}

O protocolo de estudo foi aprovado pelo Comitê de Ética em Pesquisa Envolvendo Seres Humanos sob o $n^{\circ}$ 499/04 e todos os pacientes, ou responsáveis legais, concordaram em participar do estudo, assinando o Ter- mo de Consentimento Livre e Esclarecido, após terem sido informados, minuciosamente, sobre o conteúdo e a forma do mesmo.

O tamanho amostral foi determinado previamente ao início do estudo, através de testes estatísticos específicos, levando-se em consideração os riscos $\alpha$ (5\%) e $\beta$ (20\%), bem como a variabilidade das variáveis, chegando-se a um valor mínimo de 12 indivíduos por grupo.

Foram selecionados, inicialmente, 31 pacientes com diagnóstico de PT, que deram entrada no Serviço de Ortopedia e Traumatologia do Hospital Regional de Coari - Coari/AM (HRC) e no Departamento de Ortopedia e Traumatologia da Faculdade de Medicina de Marília/ Famema - Marília/SP, no período de fevereiro de 2004 a fevereiro de 2008. Os critérios de inclusão foram: idade mínima de 10 anos e ser portador de PT no estágio II de Chiedozi. Foram excluídos pacientes que estivessem fazendo, ou tivessem feito, uso de antibióticos ou de qualquer medicação com atuação, ou efeitos colaterais, sobre o sistema imunológico com, no mínimo, 90 dias de antecedência. Seis pacientes foram excluídos desde estudo: um paciente em uso de penicilina benzatina, devido a quadro de erisipela em membros inferiores; um paciente, de origem indígena, que optou por não seguir o tratamento proposto e quatro pacientes classificados como estágio I de Chiedozi. Dessa maneira, foram incluídos 25 pacientes no total. Todos os pacientes, após o diagnóstico inicial de PT, foram submetidos a exames complementares de ultrassonografia, hemograma (HMG), hemocultura (HC) e velocidade de hemossedimentação (VHS). Alguns pacientes foram submetidos a exames de ressonância magnética, apenas para documentação dos casos. Após os exames iniciais, os pacientes foram randomizados, pelo método de sorteio de envelopes opacos e lacrados, em dois grupos: grupo A $(n=13)$ tratados com antibiótico (oxacilina, IV, 8g/ dia) ${ }^{(25)}$ e drenagem cirúrgica aberta dos abscessos; e grupo B ( $\mathrm{n}=12)$, tratados com antibiótico (oxacilina, IV, 8g/dia) e drenagem percutânea dos abscessos. Todos os pacientes foram operados pela mesma equipe cirúrgica dentro de, no máximo, 24 horas após o início da antibioticoterapia, sempre sob as mesmas condições pré e pós-operatórias (Figuras 1 a 9).

O procedimento anestésico utilizado foi do tipo bloqueio regional, raquianestesia ou geral, dependendo da musculatura envolvida. Os pacientes do grupo A, após assepsia com polivinilpirrolidona-iodo degermante, antissepsia com polivinilpirrolidona-iodo tintura e coloca- 


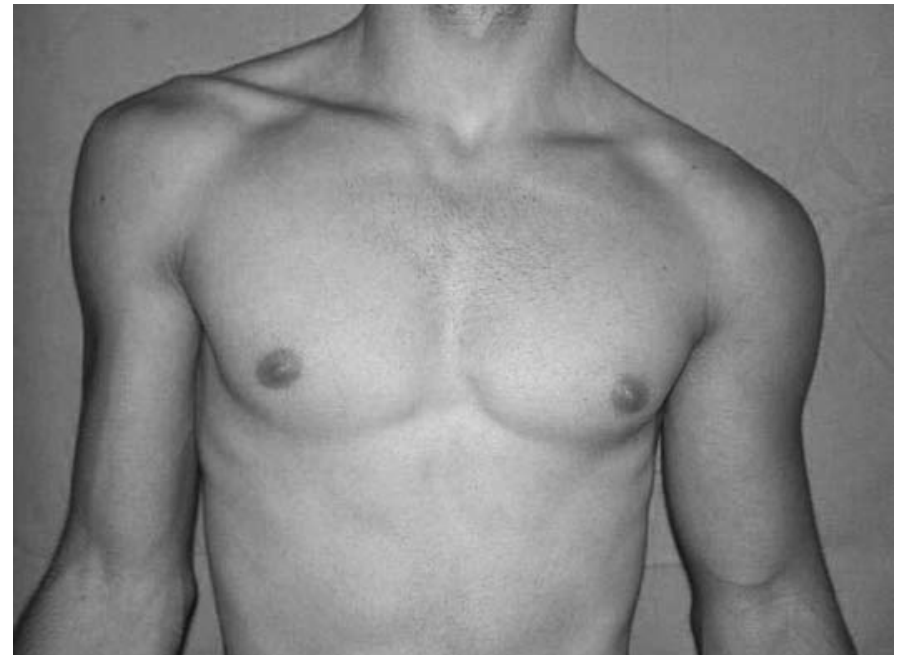

Figura 1 - Vista frontal de paciente com PT no músculo deltoide esquerdo

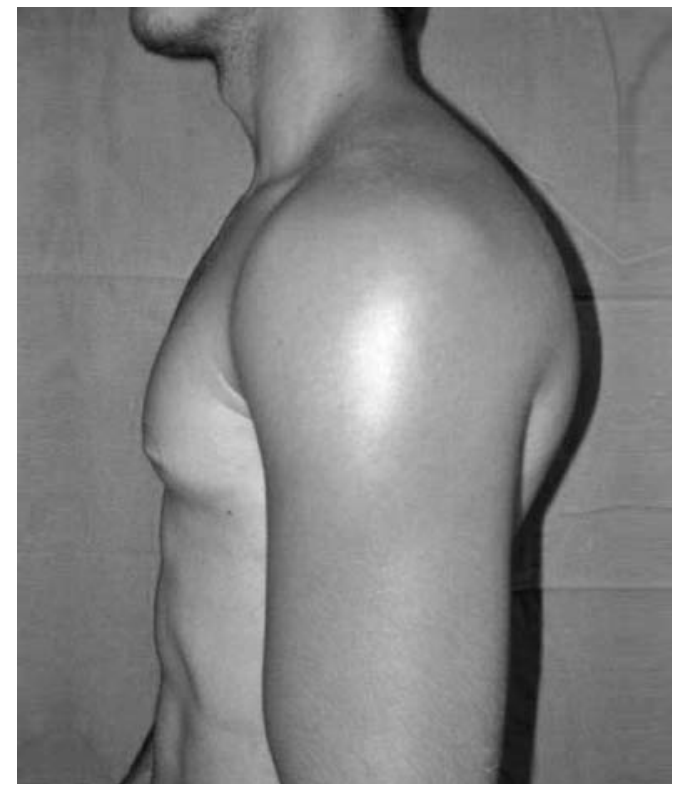

Figura 2 - Vista lateral de paciente com PT no músculo deltoide esquerdo

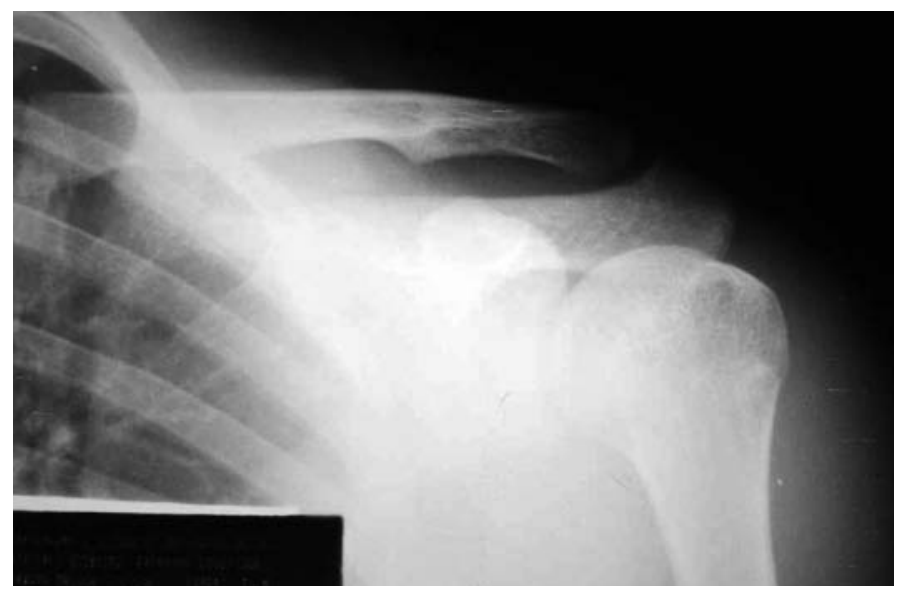

Figura 3 - Radiografia (anteroposterior) de paciente com PT no músculo deltoide esquerdo, evidenciando aumento de partes moles

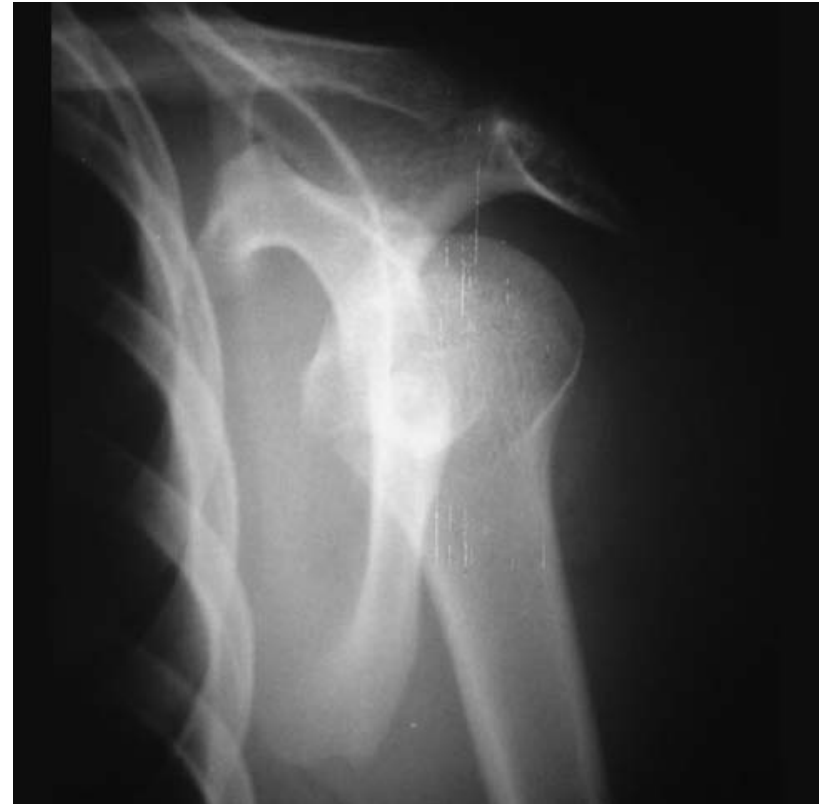

Figura 4 - Radiografia de ombro esquerdo (lateral) de paciente com PT no músculo deltoide esquerdo

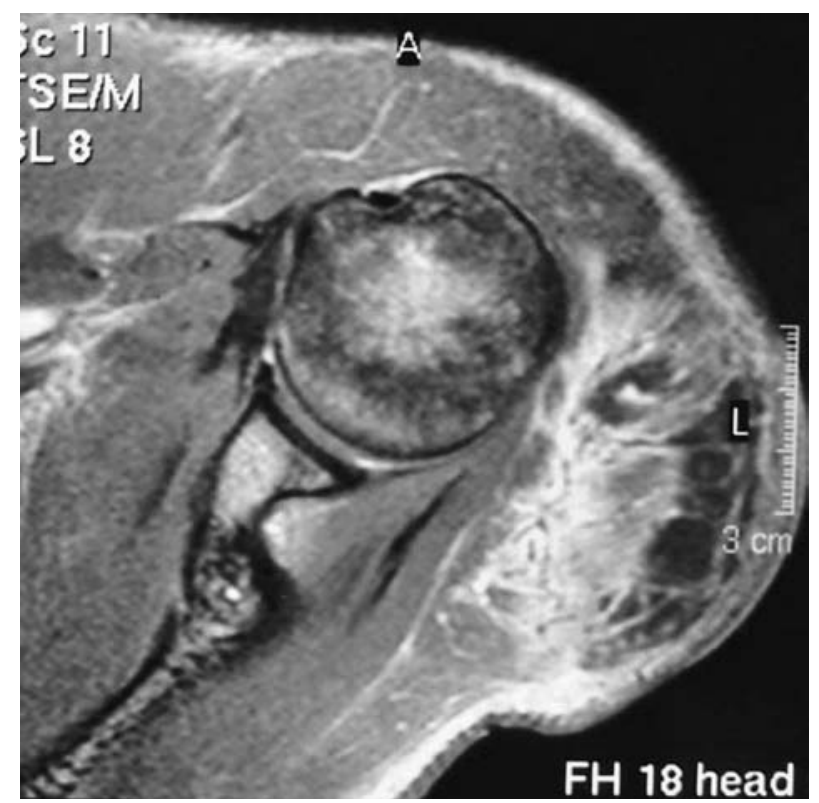

Figura 5 - Ressonância magnética de ombro esquerdo (corte transversal) evidenciando grande infiltrado no músculo deltoide

ção de campos estéreis, foram submetidos à incisão de todos os planos cirúrgicos até a abertura e drenagem dos abscessos, quando então era realizada extensa limpeza e irrigação da área cruenta com soro fisiológico 0,9\%. Não foram utilizados drenos em nenhum dos grupos. Uma vez limpos os abscessos, os planos musculares foram aproximados e a sutura do plano celular subcutâneo foi realizada com fio absorvível tipo Vicryl ${ }^{*}$ 2-0 $\left(\right.$ Ethicon $\left.^{\circledR}\right)$. A sutura da pele foi realizada com fio Mononylon ${ }^{*}$ 4-0 $\left(\right.$ Ethicon $\left.^{\circledR}\right)$. Os curativos eram abertos e 


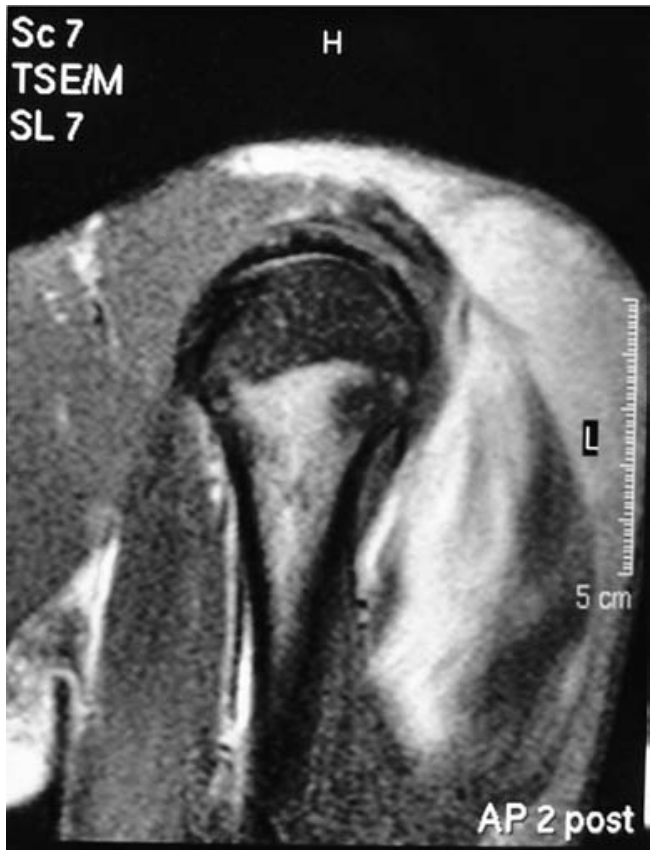

Figura 6 - Ressonância magnética de ombro esquerdo (corte coronal) de paciente com PT no músculo deltoide esquerdo

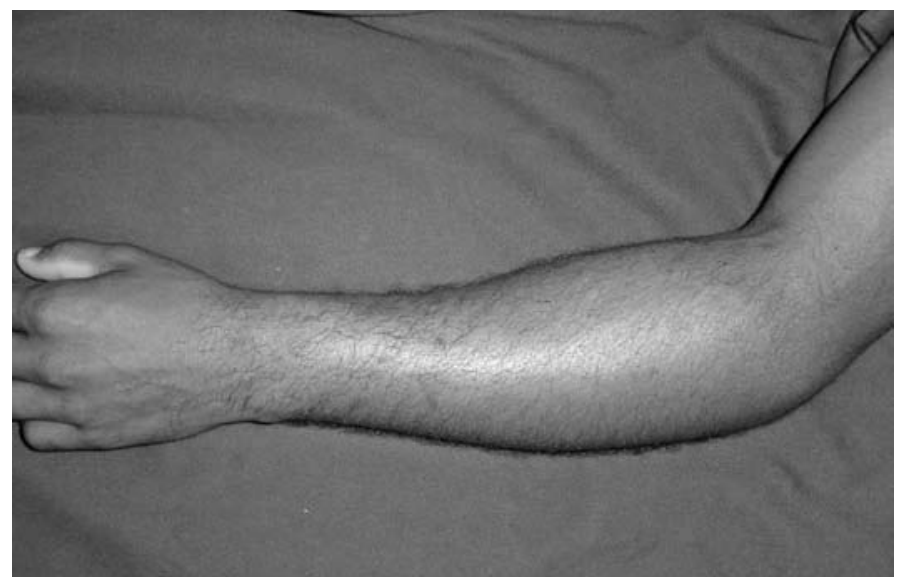

Figura 7 - Vista posterior do antebraço de paciente com PT na musculatura extensora

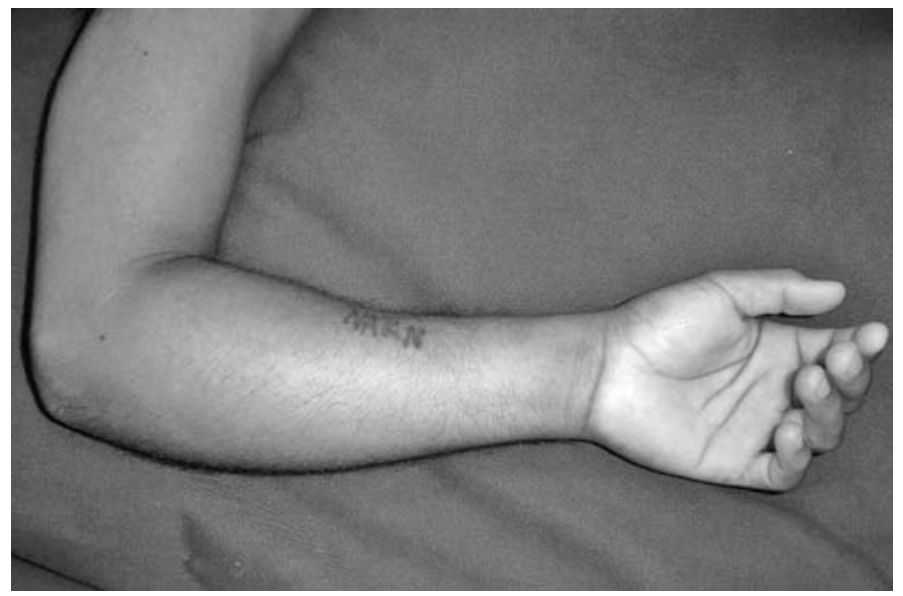

Figura 8 - Vista anterior de paciente com PT na musculatura extensora do antebraço

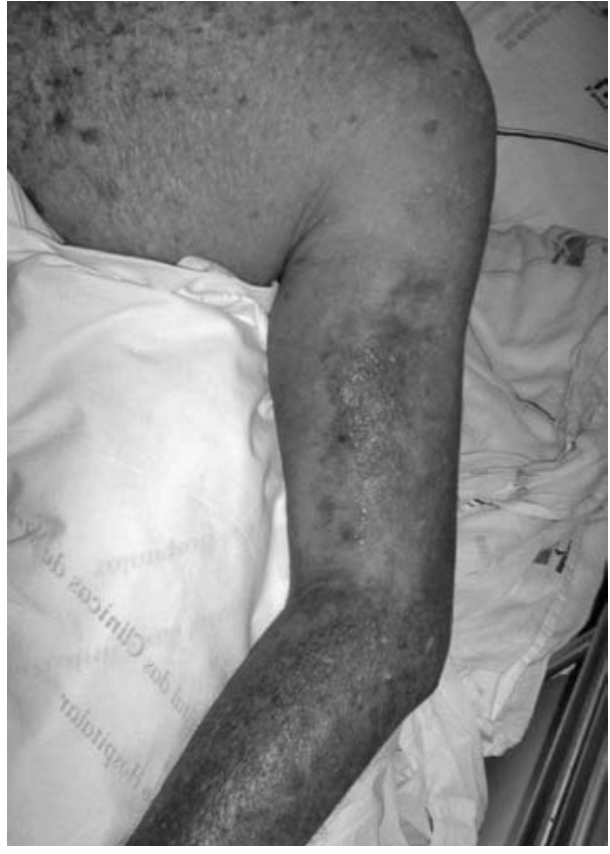

Figura 9 - Vista de paciente com PT no músculo bíceps esquerdo, com extenso edema e sufusões hemorrágicas

trocados diariamente até o sétimo dia pós-operatório; a partir de então, eram deixados abertos até o $14^{\circ}$ dia pósoperatório, quando então eram retirados os pontos.

Os pacientes do grupo B, após os mesmos procedimentos de anestesia, assepsia e antissepsia, tiveram os abscessos aspirados, percutaneamente, com cateter Jelco $^{\circledR}, n^{\circ} 14$, dirigidos por ultrassonografia (Logiq $9^{\circledR}$, GE Healthcare), após a cobertura dos transdutores ultrassonográficos com sacos plásticos estéreis em toda a sua extensão. Após a drenagem de toda a loja infecciosa, além do curativo oclusivo, não foram realizados quaisquer tipos de sutura. Padronizou-se, para todos os pacientes, a substituição de seus cateteres venosos a cada 48 horas.

O tempo de antibioticoterapia, assim como a alta hospitalar, foram condicionados à evolução satisfatória dos pacientes, sob o ponto de vista clínico (ausência de febre, dor, edema, hiperemia e calor local) e laboratorial (VHS $\leq 20 \mathrm{~mm} / \mathrm{h} ; \mathrm{HC}$ : negativa; HMG: ausência de leucocitose ou de desvios à esquerda).

\section{METODOLOGIA ESTATÍSTICA}

As variáveis: idade, sexo, tipo de microorganismo, musculatura envolvida, tempo de internação hospitalar e tempo de antibioticoterapia, foram analisadas através de testes estatísticos descritivos, paramétricos ou nãoparamétricos, no modelo inteiramente casualizado, uti- 
lizando-se, para tanto, os programas SigmaStat ${ }^{\circledR}$ versão 3.5 (Systat Software Inc., 2006) e Minitab ${ }^{\circledR}$ versão 15 (Minitab Inc., 2007). Foram calculados os valores das médias, desvios padrão, medianas, frequências, percentuais e intervalos de confiança (IC) de 95\% ( $\alpha=5 \%$ ). Convencionou-se o poder de $80 \%(\beta=20 \%)$ para todos os testes estatísticos, sendo consideradas significantes as diferenças com $p \leq 0,05$.

\section{RESULTADOS}

A idade média entre os grupos A e B foi comparada através do teste $t$ de Student, tendo sido verificada, para o grupo A, a média de 35,3 $\pm 19,2$ anos (mediana: 31 anos; IC 95\%: 24,8 - 45,8 anos) e 30,1 \pm 9 anos (mediana: 29 anos; IC 95\%: 25 - 35,2 anos) para o grupo $\mathrm{B}, \operatorname{com} p=0,41$ (Figura 10).

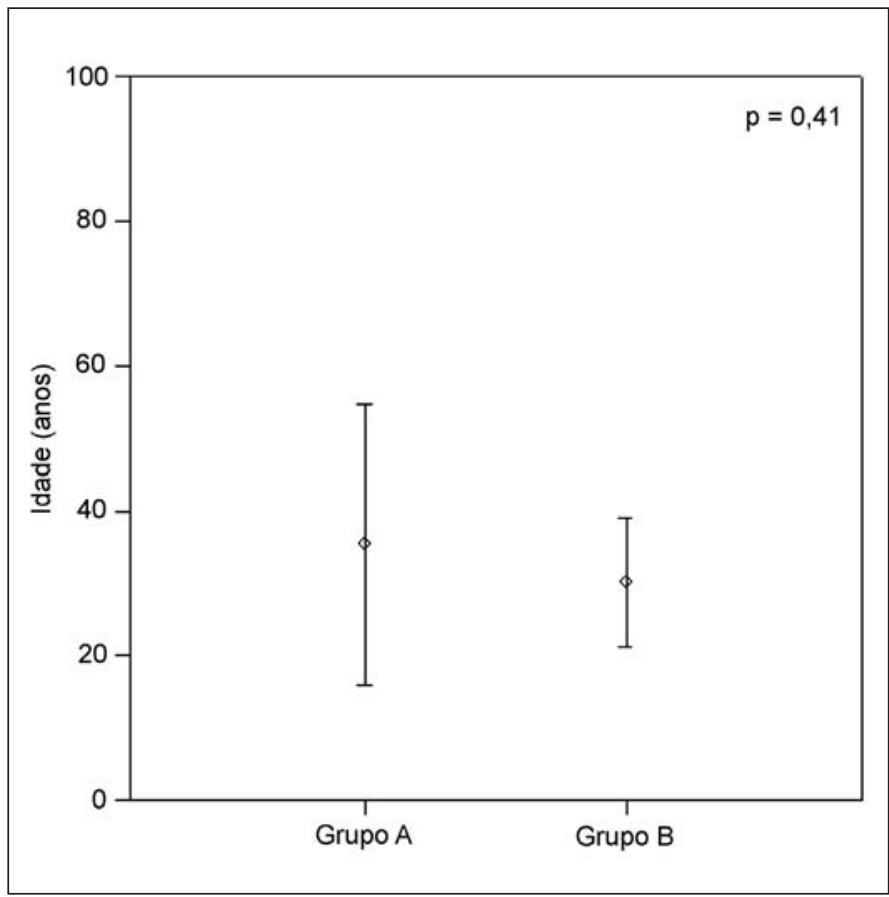

Figura 10 - Média de idade (anos) dos grupos A e B (teste $t$ de Student, $p=0,41$ )

No grupo A, oito pacientes eram do sexo feminino $(61,5 \%)$ e cinco do sexo masculino (38,5\%); no grupo $\mathrm{B}$, três eram do sexo feminino (25\%) e nove do sexo masculino (75\%). Através do teste exato de Fisher, a diferença entre as frequências dos sexos nos dois grupos foi considerada não-significante $(p=0,11)$ (Tabela 1$)$.

Com relação ao agente etiológico, no grupo A, os mais frequentes foram: 10 Staphylococcus aureus (77\%), um Streptococcus $(7,7 \%)$ e dois não identificados (15,3\%). Através do teste $Z$ para proporções, verificou-se haver
Tabela 1 - Teste exato de Fisher entre a frequência dos sexos dos grupos A e B $(p=0,11)$

\begin{tabular}{c|c|c|c}
\hline & Grupo A & Grupo B & Total \\
\hline Sexo feminino & 8 & 3 & 11 \\
\hline Sexo masculino & 5 & 9 & 14 \\
\hline Total & 13 & 12 & 25 \\
\hline
\end{tabular}

diferença significante entre as frequências dos microorganismos encontrados ( $p=0,004)$ (Figura 11). No grupo B foram encontrados: oito Staphylococcus aureus (66,7\%), três não identificados (25\%) e um Enterococcus (8,3\%). Através do teste $Z$ para proporções, verificou-se haver diferença significante entre as frequências dos microorganismos encontrados ( $p=0,04)$ (Figura 12).

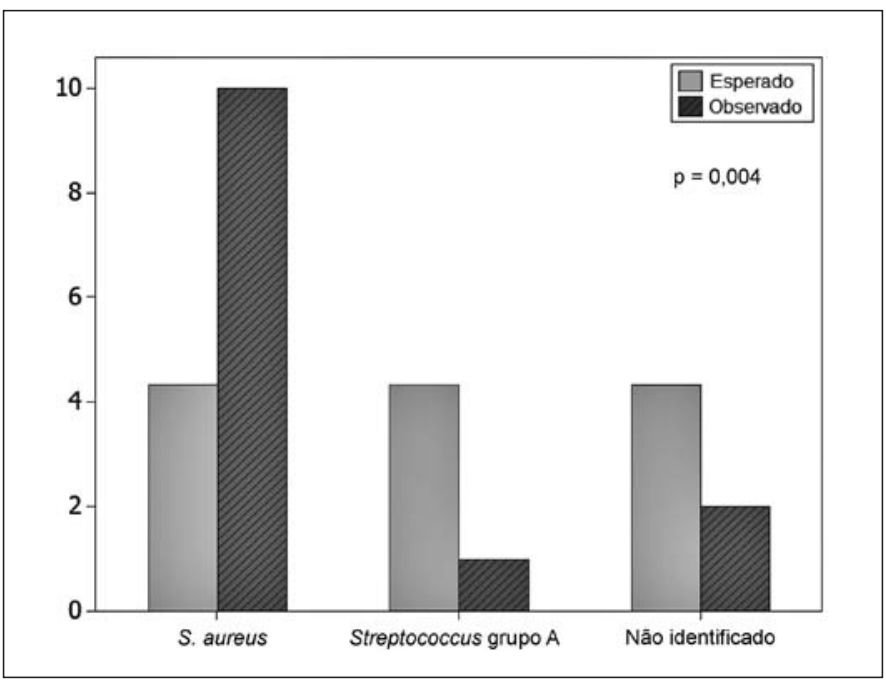

Figura 11 - Frequência dos microorganismos encontrados no grupo A (teste $Z$ para proporções, $p=0,004$ )

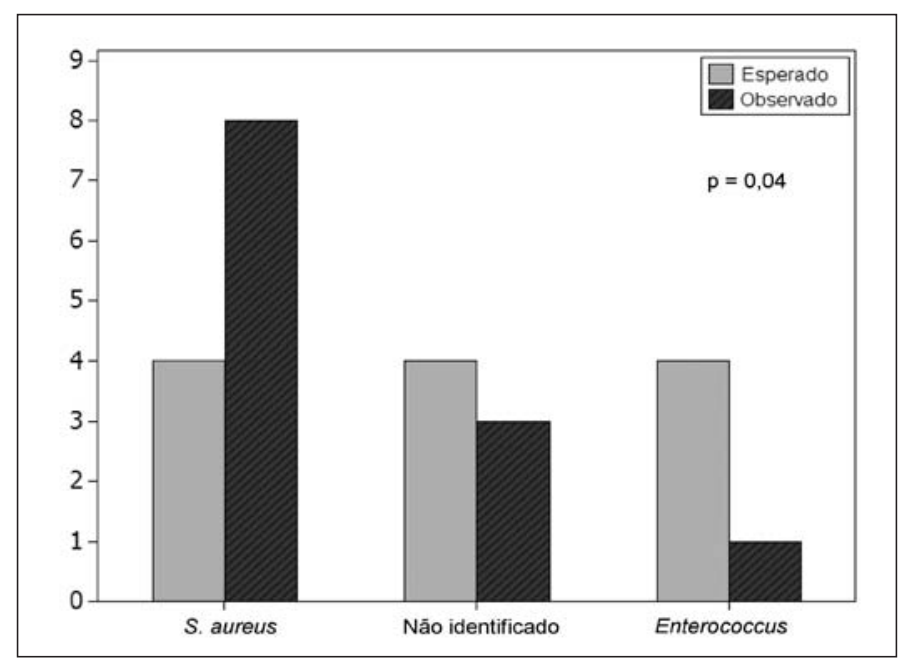

Figura 12 - Frequência dos microorganismos encontrados no grupo B (teste $Z$ para proporções, $p=0,04$ ) 
No grupo A, os músculos mais acometidos foram: três latíssimos dorsais (23\%), três extensores dos dedos e carpo (23\%), dois glúteos máximos $(15,4 \%)$, dois trapézios (15,4\%), um deltoide (7,7\%), um bíceps $(7,7 \%)$ e um quadríceps (7,7\%). Aplicando o teste $Z$ para proporções, verificou-se não haver diferença significante entre as frequências dos músculos acometidos $(p=0,85)$. No grupo $\mathrm{B}$, os músculos mais afetados foram: três deltoides (25\%), três quadríceps (25\%), um extensor dos dedos e carpo (16,7\%), dois tibiais anteriores (16,6\%), um latíssimo dorsal (8,3\%), um gastrocnêmio (8,3\%) e um flexor dos dedos e carpo (8,3\%). Aplicando o teste $Z$ para proporções, verificou-se não haver diferença significante entre as frequências dos músculos acometidos $(p=0,78)$.

O tempo médio de uso de antibiótico entre os grupos A e B foi comparado através do teste $t$ de Student, tendo sido verificada, para o grupo $\mathrm{A}$, a média de 12,2 $\pm 2,3$ dias (mediana: 12 dias; IC 95\%: 24,8 - 45,8 dias) e de $10,1 \pm 1,5$ dias (mediana: 10 dias; IC 95\%: $25-35,2$ dias) para o grupo $\mathrm{B}$, com $p=0,02$ (Figura 13).

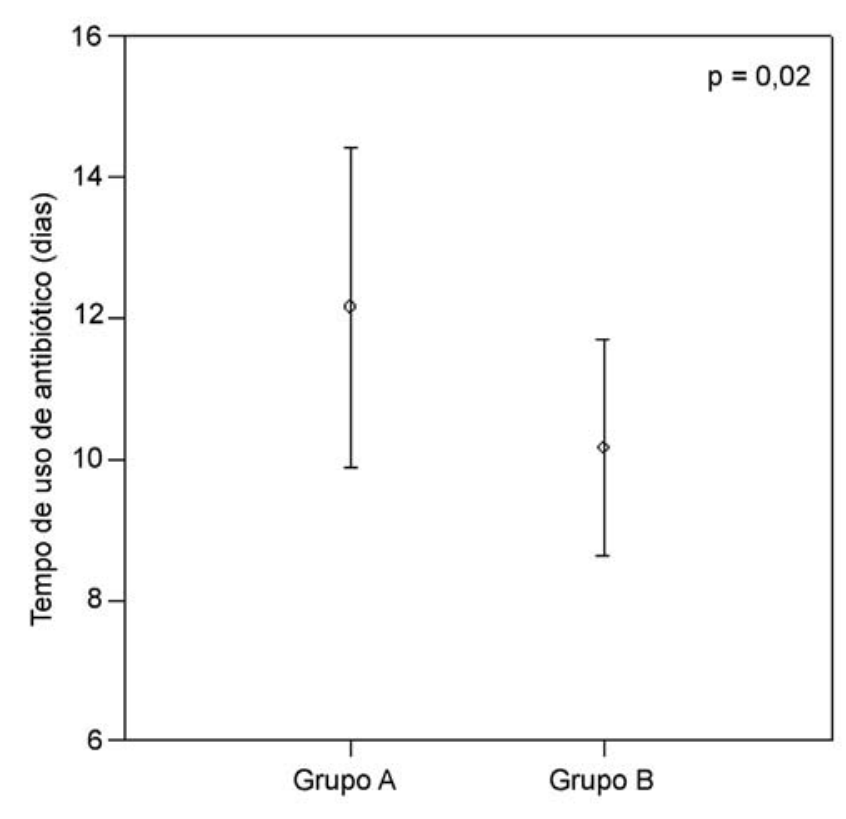

Figura 13 - Média do tempo de uso de antibióticos (dias) dos grupos A e B (teste $t$ de Student, $p=0,02$ )

Ao se comparar a idade e o tempo de antibioticoterapia no grupo A, o teste de correlação de Spearman mostrou não haver relação significante entre estas variáveis ( $p=0,64 ; \mathrm{r}=0,14$ ) (Figura 14). No grupo $\mathrm{B}$, ao se comparar a idade e o tempo de antibioticoterapia, o teste de correlação de Spearman mostrou não haver relação entre tais variáveis $(p=0,78 ; \mathrm{r}=-0,08)$ (Figura 15).

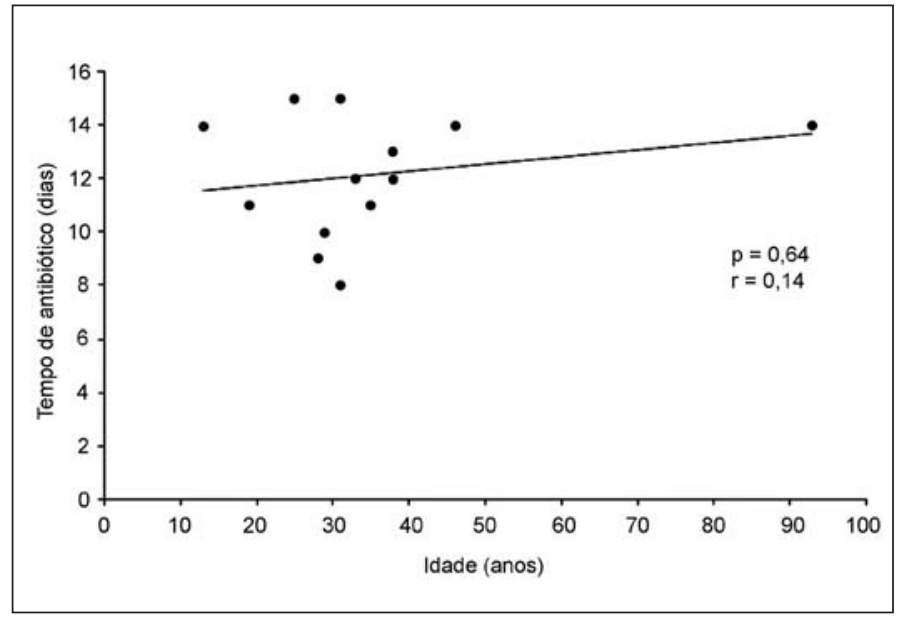

Figura 14 - Dispersão entre a idade (anos) e o tempo de uso de antibiótico dos pacientes do grupo A (teste de correlação de Spearman, $p=0,64, r=0,14$ )

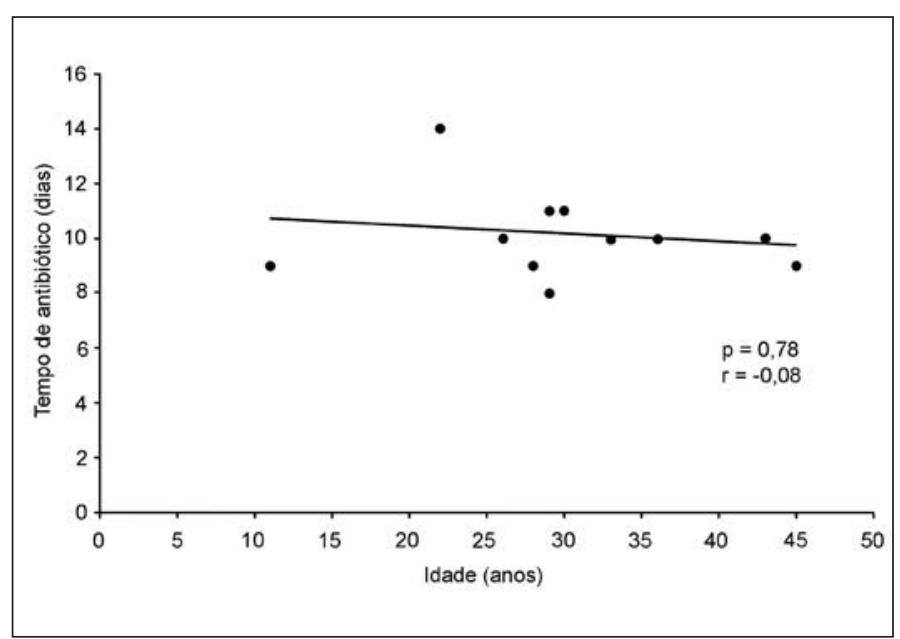

Figura 15 - Dispersão entre a idade (anos) e o tempo de uso de antibiótico dos pacientes do grupo $B$ (teste de correlação de Spearman, $p=0,78, r=-0,08$ )

Ao se comparar o tempo médio de uso de antibiótico entre os sexos, nos grupos A e B, verificou-se, no grupo $\mathrm{A}$, que a média do tempo de antibioticoterapia para o sexo feminino foi de 12,1 $\pm 2,3$ dias (mediana: 12,5 dias; IC 95\%: 10,5 - 13,8 dias) e de 12,2 $\pm 2,4$ dias (mediana: 12 dias; IC 95\%: 10,1 - 14,3 dias) para o sexo masculino. No grupo $B$, foi verificada, para o sexo feminino, a média de $10 \pm 1$ dia (mediana: 10 dias; IC 95\%: 8,9-11,1 dias) e de 10,2 $\pm 1,7$ dias (mediana: 10 dias; IC 95\%: 9,1 - 11,3 dias) para o sexo masculino. Através do teste de análise múltipla de variâncias (Anova), verificou-se não haver diferença significante entre os grupos $(p=0,15)$ (Figura 16).

O tempo de internação hospitalar entre os grupos A e B também foi levado em consideração durante a análise estatística. Através do teste $t$ de Student, verificou-se, 


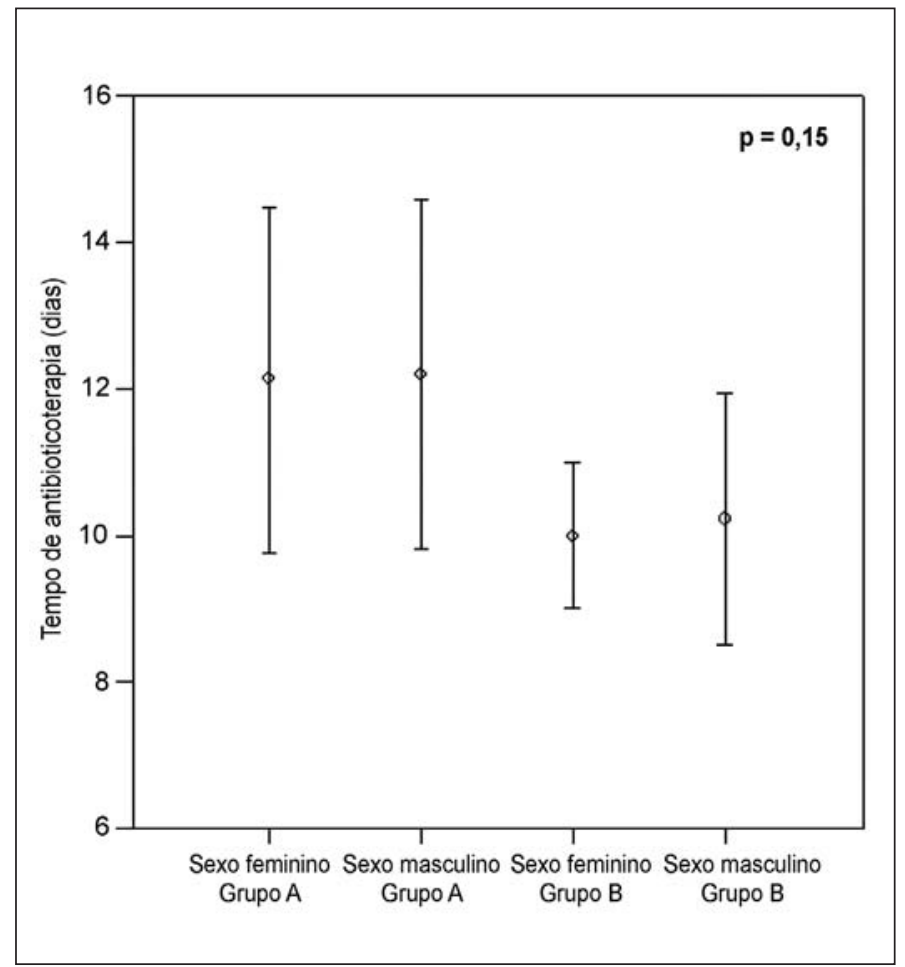

Figura 16 - Médias do tempo de uso de antibióticos (dias) dos sexos masculino e feminino dos grupos A e B (Anova, $p=0,15$ )

para o grupo A, a média de $12,7 \pm 2,1$ dias (mediana: 14 dias; IC 95\%: 11,7 - 13,9 dias) e, para o grupo B, a média de 10,6 \pm 1,6 dias (mediana: 10 dias; IC 95\%: 7,2 - 11,6 dias), com $p=0,01$ (Figura 17).

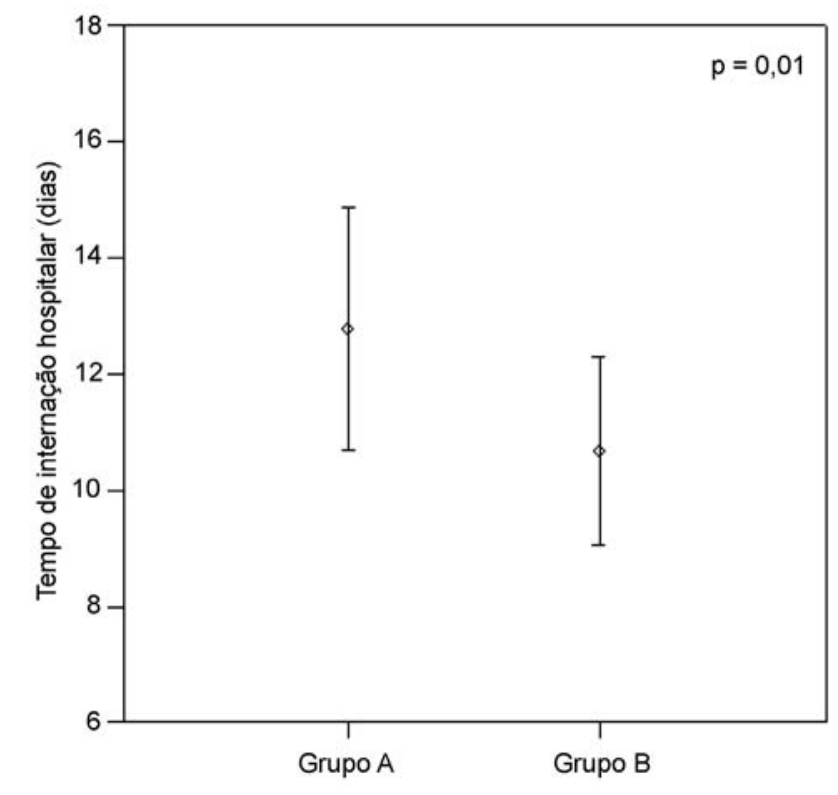

Figura 17 - Médias do tempo de internação (dias) dos grupos A e B (teste $t$ de Student, $p=0,01$ )
No grupo A, ao se comparar o tempo de internação e a idade, o teste de regressão de Spearman mostrou não haver correlação entre tais variáveis ( $p=0,72 ; \mathrm{r}=0,11)$ (Figura 18). O mesmo se deu com relação ao grupo B que, através do teste de regressão de Spearman, também mostrou não haver correlação entre tais variáveis ( $p=$ 0,$45 ; r=-0,23$ ) (Figura 19).

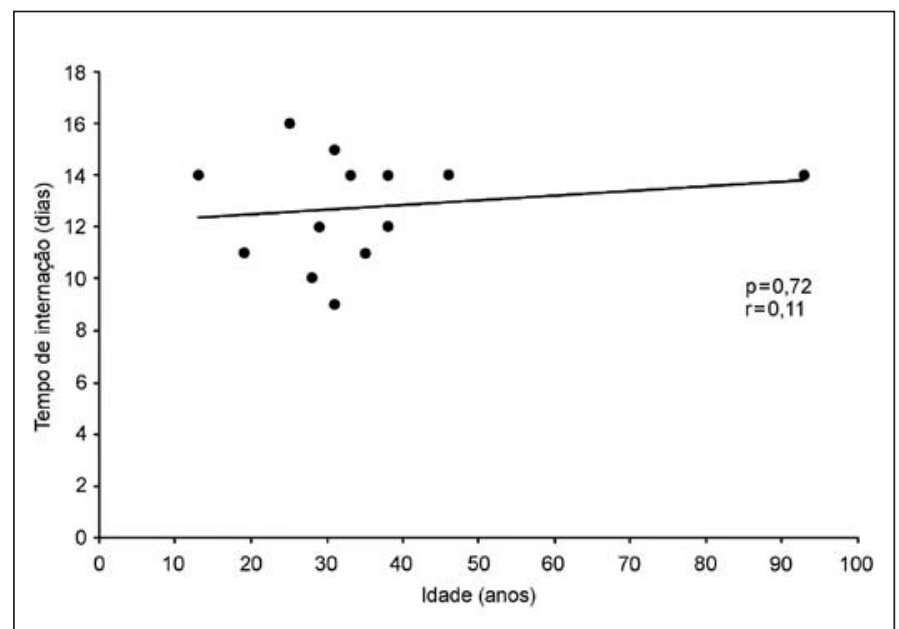

Figura 18 - Dispersão entre a idade (anos) e o tempo de internação dos pacientes do grupo A (teste de correlação de Spearman, $p=0,72, r=0,11$ )

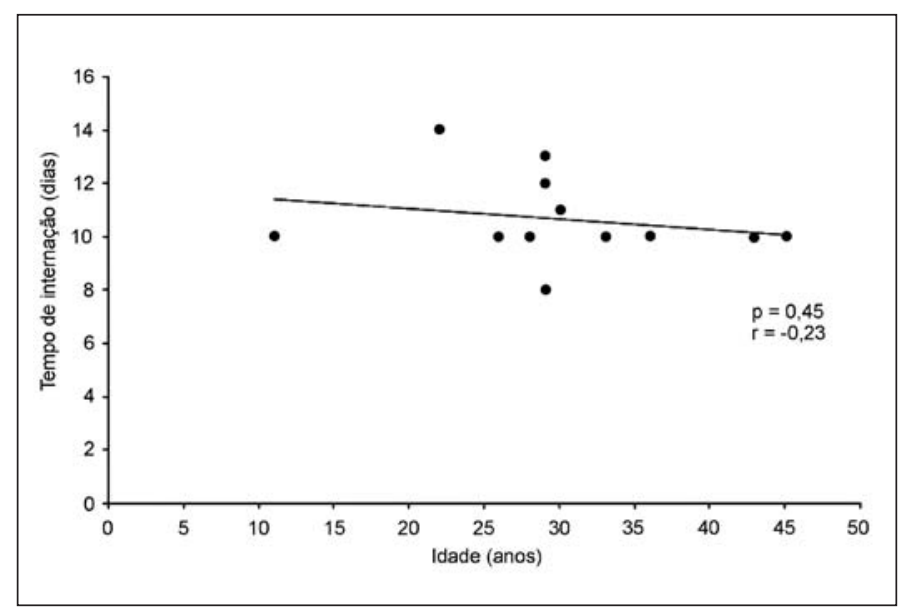

Figura 19 - Dispersão entre a idade (anos) e o tempo de internação dos pacientes do grupo B (teste de correlação de Spearman, $p=0,45, r=-0,23$ )

Ao se comparar o tempo de hospitalização entre os sexos, nos grupos A e B, verificou-se, no grupo A, que a média do tempo de internação hospitalar, para o sexo feminino, foi de 12,9 \pm 2 dias (mediana: 14 dias; IC 95\%: 11,5 - 14,3 dias) e de 12,6 $\pm 2,4$ dias (mediana: 12 dias; IC 95\%: 10,5 - 14,7 dias) para o sexo masculino. No grupo $\mathrm{B}$, foi verificada, para o sexo feminino, a média de 10,3 \pm 0,6 dias (mediana: 10 dias; IC 95\%: 9,7 - 11 
dias) e de 10,8 $\pm 1,8$ dias (mediana: 10 dias; IC 95\%: 9,5 - 12 dias) para o sexo masculino. Através do teste de análise múltipla de variâncias (Anova), verificou-se não haver diferença significante entre os grupos $(p=$ 0,09) (Figura 20).

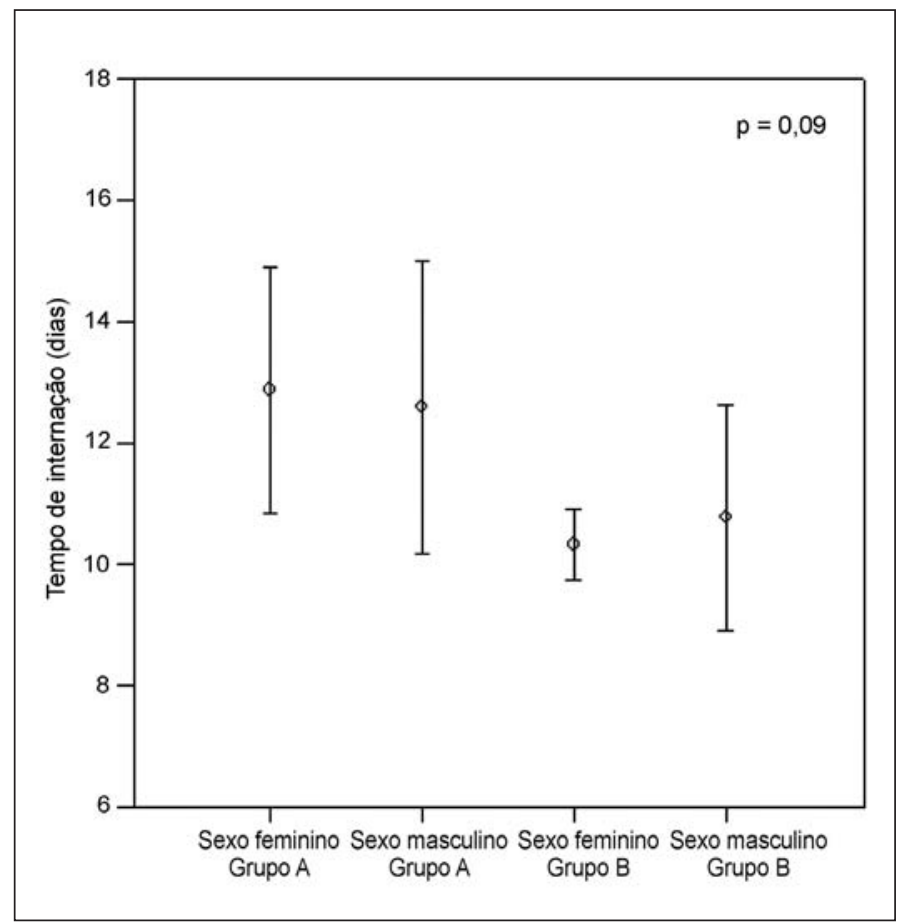

Figura 20 - Médias do tempo de internação (dias) dos sexos masculino e feminino dos grupos A e B (Anova, $p=0,09)$

Não houve complicações, pré ou pós-cirúrgicas, de qualquer tipo, em nenhum dos pacientes dos dois grupos.

\section{DISCUSSÃO}

A piomiosite tropical (PT), enfermidade que pode levar a graves complicações não somente musculoesqueléticas, mas também sistêmicas e ao óbito, apresenta grande incidência em países de clima tropical. Preocupantemente, esta enfermidade está sendo cada vez mais frequentemente relatada em países de climas temperados.

Muito embora alguns autores afirmem que a PT foi primeiramente descrita por Scriba em 1885 apud Otones et $a l^{(16)}$, Repáraz et al ${ }^{(19)}$ e Marques et $a l^{(21)}$. Chiedozi ${ }^{(5)}$ foi o primeiro a classificá-la em três estágios, que representam a progressão gradual da enfermidade:

- Estágio 1 - Início insidioso com adormecimento, formigamento, dor muscular progressiva associada a febre baixa, algumas vezes, há sinais sistêmicos inespecíficos de infecção.
- Estágio 2 - Ocorre com 10 a 21 dias de evolução e é o estágio no qual a maioria dos pacientes procura auxílio médico. Há intenso edema, dor, eritema e calor local, sendo característica a formação de abscessos.

- Estágio 3 - Tardio, com dor severa, manifestações sistêmicas de choque séptico que requerem condutas urgentes.

Concordamos com vários autores que afirmam que o estágio II é o mais frequentemente diagnosticado $^{(2-6,13,16,19,21,23)}$. Neste estudo, no qual $100 \%$ dos pacientes pertenciam ao estágio II, os principais sintomas foram: febre, tumoração e calor local e dor.

A PT pode acometer indivíduos de qualquer faixa etária ${ }^{(1-23)}$. A média de idade neste estudo coincide com a descrita por diversos autores ${ }^{(1,2,6,7,9,12,13,17)}$, havendo predomínio da segunda e terceira décadas de vida. Verificamos que ambos os grupos são homogêneos em relação à idade $(p=0,41)$, assim como à frequência dos sexos. Torna-se de crucial importância frisar que, muito embora haja um número maior de pacientes do sexo feminino no grupo A, o teste exato de Fisher mostra não haver significado estatístico $(p=0,11)$ com relação a esta variável. Vale lembrar também que o processo de randomização adotado neste estudo, através de envelopes opacos e lacrados, é validado e adotado por grande maioria dos pesquisadores da área médica ${ }^{(26,27)}$.

$\mathrm{O}$ agente etiológico mais comumente encontrado neste estudo, em ambos os grupos, foi o Staphylococcus aureus (72\%), assim como descrito na literatura especializada ${ }^{(11,12,14,18-24)}$. Este resultado fornece suporte para o início do tratamento medicamentoso, ainda que de forma empírica, na suspeita de diagnóstico de PT.

King et al ${ }^{(11)}$, aventaram a hipótese de a PT iniciar-se com a formação de um hematoma intramuscular póstraumático que, posteriormente, seria colonizado por um episódio de bacteremia. Discordamos destes autores no que se refere à necessidade de haver contusões musculares prévias e de hematomas organizados. No presente estudo, observamos que os grandes grupos musculares do tronco e dos membros superiores foram os mais afetados, sem, contudo, haver quaisquer relatos de traumas físicos prévios.

Cremos que o fato de tais grupos musculares serem os mais solicitados na grande maioria das profissões, principalmente as braçais, seja também responsável por desencadear maior número de lesões intrínsecas e pequenos sangramentos por entre as miofibrilas, sem, necessariamente, haver contusões musculares ou ainda a formação de um hematoma organizado. 
Com relação à antibioticoterapia, muito embora a diferença entre as médias de tempo de uso de antibióticos nos dois grupos seja mínima, esta foi suficiente para adquirir um caráter estatístico significante $(p=0,02)$.

De acordo com os resultados obtidos, constatamos que o menor tempo de antibioticoterapia verificado no grupo B não está, em nenhum dos grupos, relacionado à idade ou ao sexo dos pacientes (Figuras 14, 15 e 16). Não encontramos, na escassa literatura disponível, qualquer autor que fizesse menção sobre o tempo de uso de antibiótico relacionado à idade ou ao sexo dos pacientes no tratamento da PT. Podemos, contudo, inferir que ausência de grandes incisões cirúrgicas e, consequentemente, o menor dano tecidual dos envelopes de partes moles, nos pacientes submetidos à drenagem percutânea dos abscessos, contribuíram para o menor tempo de utilização de antibióticos.

Uma clara diminuição do tempo de internação hospitalar foi também verificada nos pacientes do grupo $\mathrm{B}$, quando comparados aos pacientes do grupo $\mathrm{A}$ $(p=0,01)$.

Nos resultados encontrados, muito embora haja discreta tendência em haver correlação estatística entre o período de internação e o sexo dos pacientes $(p=0,09)$, os testes realizados demonstram que a redução do tempo de hospitalização não está relacionada com o sexo ou com a idade dos pacientes (Figuras 18, 19 e 20). Uma vez mais, não encontramos na literatura qualquer autor que referisse a possível relação entre o tempo de uso de antibiótico e a idade ou o sexo dos pacientes, em casos de PT. Deduz-se que a menor agressão tecidual, proporcionada pela drenagem percutânea dos abscessos, conferiu aos pacientes do grupo B um menor tempo de recuperação clínica e, consequentemente, alta hospitalar precoce.

A ausência de complicações pré e pós-operatórias é também relatada por diversos autores que realizaram o procedimento cirúrgico aberto clássico até o estágio 2 de Chiedozi ${ }^{(4,6,8,12,13)}$.

\section{CONCLUSÃO}

Neste estudo, a drenagem percutânea dos abscessos associada à antibioticoterapia constituiu-se um método eficaz para o tratamento da piomiosite tropical, grau II de Chiedozi, abreviando o tempo total de uso de antibiótico e de internação hospitalar dos pacientes.

\section{REFERÊNCIAS}

1. Bickels J, Ben-Sira L, Kessler A, Wientroub S. Primary pyomyositis. J Bone Joint Surg Am. 2002;84(12):2277-86.

2. Gibson RK, Rosenthal SJ, Lukert BP. Pyomyositis. Increasing recognition in temperate climates. Am J Med. 1984;77(4):768-72.

3. Kallen P, Nies KM, Louie JS, Keller M, Worthen N, Bayer AS. Tropical pyomyositis. Arthritis Rheum. 1982;25(1):107-10.

4. Hall RL, Callaghan JJ, Moloney E, Martinez S, Harrelson JM. Pyomyositis in a temperate climate. Presentation, diagnosis, and treatment. J Bone Joint Surg Am. 1990;72(8):1240-4.

5. Chiedozi LC. Pyomyositis. Review of 205 cases in 112 patients. Am J Surg. 1979;137(2):255-9.

6. Marques GC, Mauro Filho GL, Valiatti M, Hamra A, Valiatti J, Mastrocola Jr A, et al. Piomiosite tropical: estudo retrospectivo de 27 casos. Rev Bras Reumatol. 1995;35(4):193-200.

7. Peckett WR, Butler-Manuel A, Apthorp LA. Pyomyositis of the iliacus muscle in a child. J Bone Joint Surg Br. 2001;83(1):103-5.

8. Talavera MB, Wakai M, Campos LMA, Baldacci ER, Silva CAA. Piomiosite bacteriana aguda (PBA) em crianças eutróficas. Rev Bras Reumatol. 2003;43(4):259-64.

9. Azevedo PS, Matsui M, Matsubara LS, Paiva SAR, Inoue RMT, Okoshi MP, et al. Piomiosite tropical: apresentações atípicas. Rev Soc Bras Med Trop. 2004;37(3):273-8.

10. Braz AS, Fernandes JMC, Couto RAL, Szajubok JCM, Moura Junior JE, Chahade WH. Piomiosite tropical: descrição de quatro casos. Rev Bras Reumatol. 2000;40(2):81-6.

11. King RJ, Laugharne D, Kerslake RW, Holdsworth BJ. Primary obturator pyomyositis: a diagnostic challenge. J Bone Joint Surg Br. 2003;85(6):895-8.

12. Villamil-Cajoto I, Maceiras-Pan F, Villacián-Vicedo MJ. Piomiositis: presentación de 17 casos em niños y adultos. Rev Med Chile. 2006;134(1):31-8.

13. Fujiki EN, Milani C, Fukushima WY, Mader AM, Nascimento FP, Furlan C, et al. Piomiosite tropical. Arq Med ABC. 2005;30(1):48-53.

14. Takayasu V, Onuchic MH, de Campos FP, de Albuquerque RP. [Tropical myositis]. Rev Hosp Clin Fac Med Sao Paulo. 1993;48(3):112-5.

15. Pereira FO, Medeiros YS. [Painful syndromes in diabetic patients due to skeletal muscle injuries]. Arq Bras Endocrinol Metabol. 2006;50(5):957-62.

16. Otones LL, Román SV, Martín Gí, Conejo PR, Tomé MIG. Piomiositis en los niños: no solo una enfermedad tropical. An Pediatr (Barc). 2007;67(6):578-81.

17. Ozkan K, Unay K, Ugutmen E, Eren A, Eceviz E, Saygý B. Pyomyositis of tensor fascia lata: a case report. J Med Case Reports. 2008;2:236.

18. Tanir G, Tonbul A, Tuygun N, Aydemir C, Ertan U. Soft tissue infections in children: a retrospective analysis of 242 hospitalized patients. Jpn J Infect Dis. 2006;59(4):258-60.

19. Repáraz FJ, Repáraz J, Martínez Bayarri M, Tejero A, Corchuelo C, Avila A. [Primary pyomyositis caused by Streptococcus intermedius]. An Sist Sanit Navar. 2007;30(2):273-9.

20. Kamal T, Hall M, Moharam A, Sharr M, Walczak J. Gluteal pyomyositis in a non-tropical region as a rare cause of sciatic nerve compression: a case report. J Med Case Reports. 2008;2:204.

21. Marques GC, Marques MN, Miyoshi SR, Maciel RR, Macchione MC. Artrites sépticas e piomiosite tropical. Temas Reumatol Clin. 2001;2(4):5.

22. Ruiz ME, Yohannes S, Wladyka CG. Pyomyositis caused by methicillin-resistant Staphylococcus aureus. N Engl J Med. 2005;352(14):1488-9.

23. García-Mata S, Hidalgo A, Esparza J. [Primary pyomyositis of the psoas muscles in a temperate climate. Review of two cases in children followed up over the long term]. An Sist Sanit Navar. 2006;29(3):419-31.

24. Tong CW, Griffith JF, Lam TP, Cheng JC. The conservative management of acute pyogenic iliopsoas abscess in children. J Bone Joint Surg Br. 1998;80(1):83-5.

25. Gilbert DN, Moellering RC Jr, Eliopoulos GM, Sande MA, editors. The Sanford guide to antimicrobial therapy. 38th ed. Sperryville: Sanford Editors; 2008.

26. Doig GS, Simpson F. Randomization and allocation concealment: a practical guide for researchers. J Crit Care. 2005;20(2):187-91.

27. Altman DG, Schulz KF. Statistics notes: Concealing treatment allocation in randomised trials. BMJ. 2001;323(7310):446-7. 